\title{
Testing of a Wireless Sensor System for Instrumented Thermal Protection Systems
}

\author{
Allen T. Kummer, ${ }^{1}$ Erik D. Weir, ${ }^{2}$ Trey J. Morris, ${ }^{3}$ Corey W. Friedenberger, ${ }^{4}$ Aseem Singh, ${ }^{5}$ \\ Robert M. Capuro, ${ }^{6}$ and Sven G. Bilén ${ }^{7}$ \\ The Pennsylvania State University, University Park, PA, 16802 \\ Johnny Fu ${ }^{8}$ \\ Sierra Lobo, Inc., Moffett Field, CA, 94035 \\ Gregory T. Swanson ${ }^{9}$ \\ ERC, Inc., Moffett Field, CA 94035 \\ and \\ David B. Hash ${ }^{10}$ \\ NASA Ames Research Center, Moffett Field, CA 94035
}

\begin{abstract}
Funded by NASA's Constellation Universities Institutes Project (CUIP), we have been developing and testing a system to wirelessly power and collect data from sensors on space platforms in general and, in particular, the harsh environment of spacecraft re-entry. The elimination of wires and associated failures such as chafing, sparking, ageing, and connector issues can increase reliability and design flexibility while reducing costs. These factors present an appealing case for the pursuit of wireless solutions for harsh environments, particularly for their use in space and on spacecraft. We have designed and built a prototype wireless sensor system. The system, with capabilities similar to that of a wired sensor system, was tested in NASA Ames Research Center's Aerodynamic Heating Facility and Interaction Heating Facility. This paper discusses the overall development effort, testing results, as well as future directions.
\end{abstract}

\section{Introduction}

$\mathrm{T}$ HERE exists a significant need for robust Wireless Sensor Systems (WLSS), particularly for harsh environments such as those that exist on aerospace platforms [1,2]. The purpose of any system of sensors is to gather data remotely from specific points of interest dispersed throughout a larger system. Often, however, it is difficult to make these measurements at the location of interest when it is necessary to run a physical wire to points of interest. For example, NASA engineers have identified the need for wireless sensors to ameliorate the problems associated with physically interconnected systems, specifically within spacecraft Thermal Protection Systems (TPS) utilized during re-entry [3]. For some missions, the heat shield must be ejected, which requires that wires

\footnotetext{
Graduate Student Research Assistant, Electrical Engineering, 121 EE East.

Graduate Student Research Assistant, Electrical Engineering, 121 EE East; now with Buzby Networks, State

College, PA.

Undergraduate Student, Aerospace Engineering, 229 Hammond.

Undergraduate Student, Aerospace Engineering, 229 Hammond, AIAA Student Member.

Research Assistant, Electrical Engineering, 121 EE East.

Programs Manager, Systems Design Lab, Electrical Engineering,121 EE East, AIAA Member.

Associate Professor, Electrical Engineering, 213 Hammond, AIAA Associate Fellow.

Electrical Engineer, Thermophysics Facilities Branch, MS229-4.

Electrical Engineer, Entry Systems Branch, MS230-2.

10 Deputy Branch Chief, Aerothermodynamics Branch, MS230-1, AIAA Senior Member.
}

American Institute of Aeronautics and Astronautics 
connecting the TPS sensors to the spacecraft must be severed for heat shield separation to occur; if these wires do not sever properly, then mission failure may occur. Hence, data collection on TPS can be significantly more difficult than elsewhere on a spacecraft and pits the desire for more data against the risk associated with wire severance malfunction.

Wireless solutions enable a reduction in the quantity and complexity of the physical interconnects, thus offering advantages over wired systems in several areas:

- Reduced Life Cycle Cost: Wireless systems reduce life cycle costs through ease of upgrades, reconfiguration, troubleshooting, and root cause determination [1].

- Improved Reliability: Wireless systems reduce typical wired system failure modes caused by failures of wires, insulation, and connectors [4].

- Increased Accessibility: Structural barriers limit physical access to wired subsystems.

- Mass Reduction: Wireless systems offer significant mass savings by eliminating the need for cables, brackets, connectors, bulkheads, cable trays, structural attachments, and reinforcements.

- Adaptability: Wireless systems offer significant advantages over wired systems when upgrades or reconfigurations are required.

\section{A. Problem Description}

NASA engineers have placed a high priority on the development of wireless sensor networks in order to instrument TPS as well as for use in decreasing wire failure in aerospace systems [4]. An additional need for easily configurable wireless sensor systems has been identified within testing facilities that require rapid test-bed reconfiguration such as in the Aerodynamic Heating Facility (AHF), Interaction Heating Facility (IHF), and other arc jet test facilities at NASA Ames Research Center. Developing WLSS for testing facility applications has a lower developmental risk than deploying a WLSS on a space mission, yet can advance the Technology Readiness Level (TRL) of the technology. Preliminary testing by the University of Idaho ThermaSense team demonstrated wireless connectivity in a vacuum plasma test environment [3]. The WLSS described herein was subjected to rigorous testing to verify that the system can support high-speed data collection, support multiple sensor types, and operate in the NASA Ames tests chambers in the presence of hypersonic plasma flow.

\section{B. Context}

The recent rise in wireless sensor networks (WSNs) is largely due to recent advances in CMOS technology and the development of low power wireless protocols [5]. Specifically, the advent of mesh networking has caused many companies to invest in wireless personal area networks (WPANs) for the purpose of addressing significant markets such as healthcare and industrial applications [6]. This is shown by the wide variety of WSN applications, everything from duck habitat monitoring to temperature monitoring of refrigerated trucks $[7,8]$. The work detailed in this paper addresses the important application of WSN for aerospace platforms. NASA and the U.S. military have made the problem of wire ageing a national priority in light of the downing of Swissair 111 in 1998 and of TWA 800 in 1996, both tragedies attributed to faulty wiring [1]. The technology presented by this paper furthers the goal of removing the problems that ageing wires could cause, particularly for aerospace systems with long service lifetimes.

\section{Wireless Protocol}

The wireless protocol used for the WLSS was selected to meet the communication requirements found for reentry applications of robustness, low power, and self-healing capability. The ZigBee wireless protocol, based on the IEEE 802.15.4 standard, meets these requirements and has been proven in other harsh environment wireless sensor systems [7]. The protocol supports mesh networking, in which every wireless node (or group of sensors in this case) has the potential to connect to any other node/sensor group to route its data. This feature prevents changing environmental conditions from interrupting a hard-coded router path. As more and more sensors are wirelessly incorporated into the system, the probability that these sensors will become lost, or cut off from the network, becomes insignificant due to the number of network access points. ZigBee can operate in either the 2.4-GHz band or the $900-\mathrm{MHz}$ band. The $2.4-\mathrm{GHz}$ band was selected over the $900-\mathrm{MHz}$ band because it has a higher data rate and requires a smaller antenna. 
One of the concerns about testing in an arc jet environment is the presence of broadband electromagnetic interference (EMI) due to the arcing process used to generate the plasma flow. To investigate the potential impact of this EMI, we measured the signal levels at frequencies of interest (particularly in the 2.4-GHz band). Figure 1 shows a spectrum analyzer capture while operating at the NASA Ames Research Center's IHF, which has similar testing characteristics to the AHF. The figure shows that the $2.4-\mathrm{GHz}$ band contains little noise during the operation of the facility.

\section{Test Facility Background}

We designed and built a prototype WLSS with capabilities similar to that of a wired sensor system, in the sense that it could measure several different sensor types at various sampling frequencies. This WLSS was tested first in an AHF test series in January 2010 and in an IHF test in February 2011. These facilities are used to evaluate typical heating responses of TPS materials during atmospheric entry. These facilities are ideal for testing the robustness of a wireless network because the environment approximates atmospheric entry conditions. The AHF can provide up

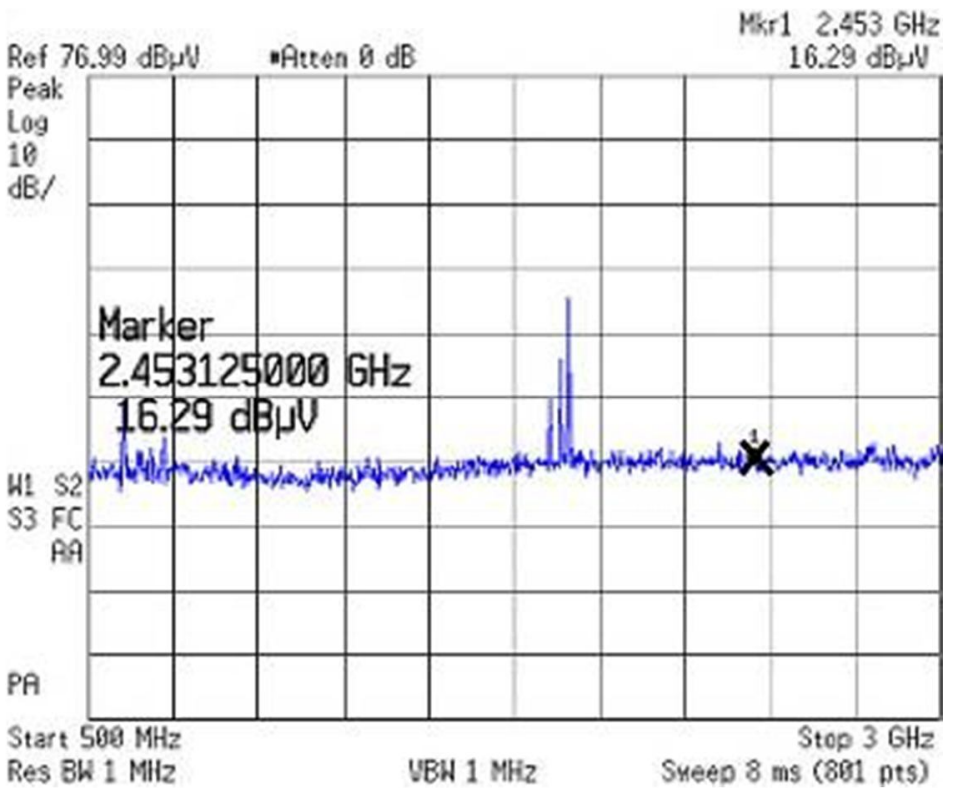

Figure 1. RF noise measurement of the NASA Ames IHF during operation where the marker denotes the transmission frequency of interest and the observed spikes at $\sim 1.8 \mathrm{GHz}$ are attributed to phone communication.

to $20 \mathrm{MW}$ of power and operate at pressures up to $1,000 \mathrm{kPa}$. In addition, the AHF has five sample insertion mechanisms (called sting arms) that can be separately controlled to allow multiple samples to be tested sequentially before breaking vacuum. This capability allowed the WLSS to piggyback with an existing test. The IHF can provide up to $60 \mathrm{MW}$ of power and operate at pressures up to $1,000 \mathrm{kPa}$. There are three sample insertion mechanisms in the IHF.

\section{WLSS System Description}

In order for the wireless system to be viable as a replacement for a wired system, certain performance parameters must be met. Working with test engineers for the AHF, the performance parameters benchmarked for the AHF test were as follows:

- The system must make a minimum data collection rate $\geq 10 \mathrm{~Hz}$ per channel;

- The system must allow for real-time data collection and viewing (at $\geq 1 \mathrm{~Hz}$ );

- The network must function in close proximity to plasma and test EMI; and

- The wireless system must be reliable and mechanically robust.

With respect to size, mass, and power, the systems needs to fit inside the TPS sample block
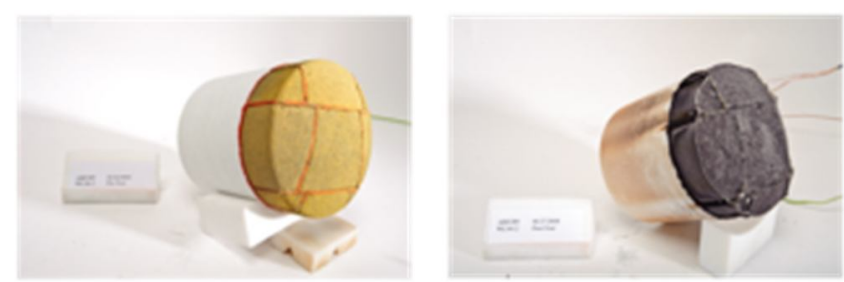

Figure 2. TPS sample pre-test (left) and post test (right).

(shown in Figure 2), mass was not critical, and power should be low enough to allow the battery to last the duration of setup, checkout, and test run. The system should point toward further refinement in miniaturization.

The system we developed is a Telegesis-based ZigBee network that can measure up to eight differential sensors at data rates up to $\sim 60 \mathrm{~Hz}$. The system collects and stores the data onboard, and sends a slower subset (which the user can select for averaging or decimation) of the collected data back to the receiver in real time at $2 \mathrm{~Hz}$. Because the system uses the ZigBee protocol, the network is robust, reliable, and self-healing. Additionally, we determined that the system is capable of operating in close proximity to the plasma. The system has a circular form factor with 
a stack of three boards measuring 2.75 inches (7.0 $\mathrm{cm})$ in diameter and a height of 0.75 inches (1.9 $\mathrm{cm}$ ), as shown in Figure 3. For this system, we did not optimize for size, rather for ease of assembly and flexibility.

\section{A. Hardware Overview}

At the onset of the development effort, functional modularity in the design was imposed as a requirement to assure the capability to modify specific functional blocks, while still maintaining core functionality. Accordingly, the functionality of the prototype system is partitioned into four subsystems. These subsystems are depicted in the functional diagram shown in Figure 4.
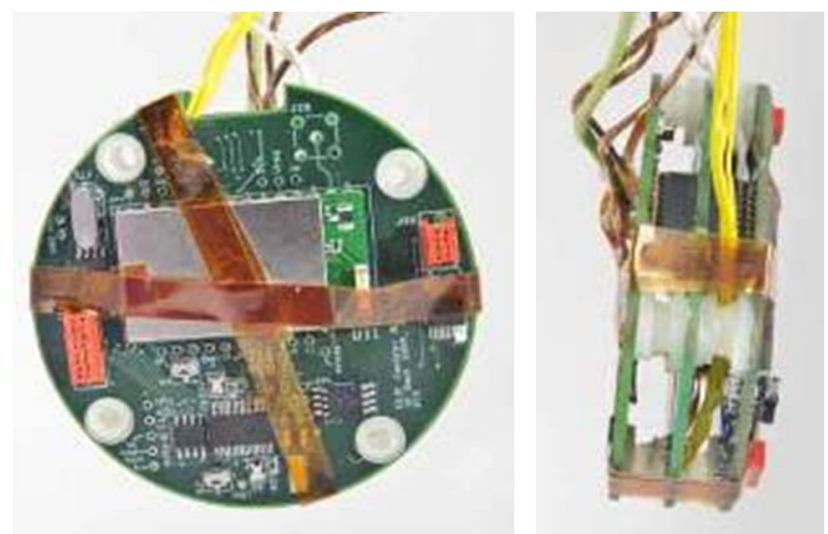

Figure 3. Top and side view of the WLSS.

The first of the WLSS subsystems is the signal conditioner, where the sensor data is converted from differential analog voltage signals to 16-bit digital data. This is accomplished through multiplexing the differential signals from the sensors through a single gain stage. This gain stage is comprised of an AD8236ARM instrumentation amplifier for gain, an LMP2231BMF operational amplifier in a SallenKey low pass filter configuration for signal noise filtration, and finally an AD7680BRM ADC to convert the analog signal into a serial digital data stream.

The second subsystem is the controller, the purpose of which is to control the data acquisition from the signal conditioning subsystem, as well as to manage the communication with the ZigBee network. The controller board reads the digital data into the PIC16F886 microcontroller, stores the data at a $60-\mathrm{Hz}$ frequency into RAM (24LC512, 32000 data points in the 512-kbit storage at 16 bits), and outputs an update signal at $2 \mathrm{~Hz}$ to allow for real-time data tracking. This data is not sent in real time over the network, however, due to limitations of the ETRX2 Telegesis module and the ZigBee 2007 (non-Pro) version that was used on the controller board. The controller subsystem also handles temperature measurements with the included IC temperature sensor to facilitate software cold junction

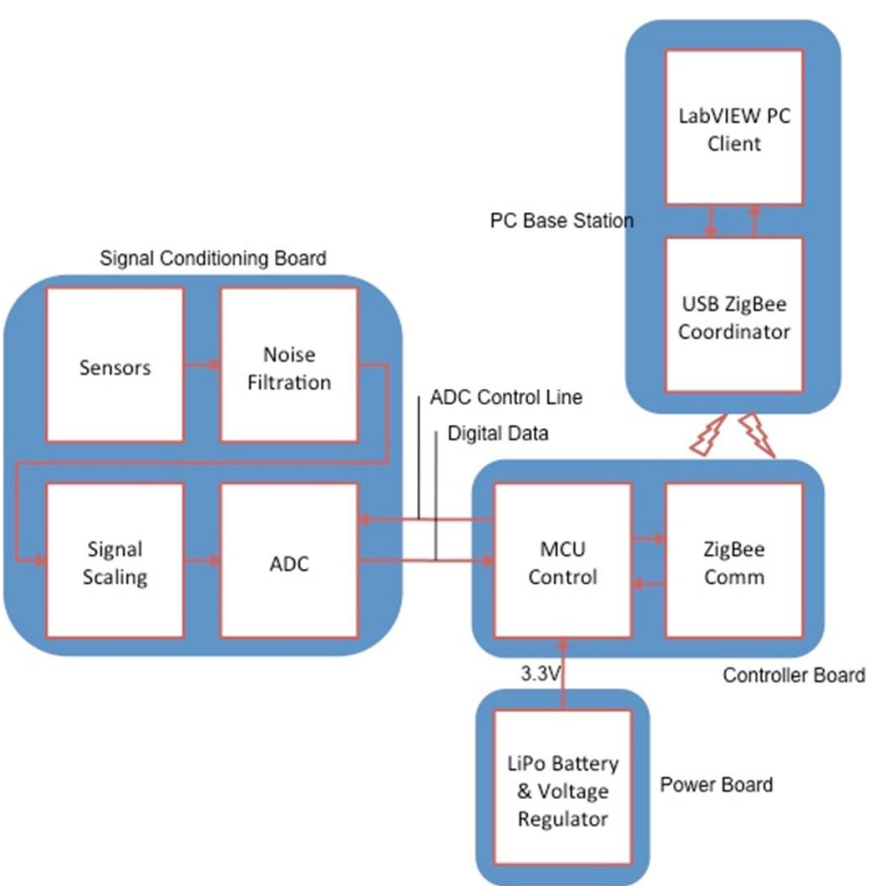

Figure 4. Subsystems of the WLSS prototype. compensation (CJC) by the base station software, which is a technique that provides accurate CJC. Microcontroller code was written using MPLAB CCS C-code.

The third subsystem is the power module, which utilizes a single-cell rechargeable Lithium Polymer (LiPo) battery that supplies $3.7 \mathrm{~V}$. This voltage is regulated to a constant $3.3 \mathrm{~V}$ by a high efficiency linear regulator. A Zener diode provides reverse polarity and overvoltage protection. In addition, an onboard optional constant current source can be used in the event that a sensor requires external stimulation, i.e., is not passive power. In this configuration, which was not optimized for power consumption, the battery can provide about 4 hrs of operation.

The fourth subsystem is the base station controller, which consists of a USB ZigBee coordinator dongle connected to a PC. The receive antenna is part of the dongle. The data acquisition software on the PC was written within the National Instruments LabVIEW suite. 


\section{Test Results}

The WLSS system, with capabilities similar to that of a wired sensor system, was tested in the NASA Ames Research Center's Aerodynamic Heating Facility (AHF) in January 2010 and Interaction Heating Facility (IHF) in February 2011. A description of these two tests and the results are provided below.

\section{A. AHF Test Description}

The high frequency data-gathering test was performed in the AHF on 10 January 2010. For this test a WLSS package (refer to Figures 2 and 3) was placed onto Sting Arm 1 and inserted into the jet for targeted heating rate of $170 \mathrm{~W} / \mathrm{cm}^{2}$ for 60 seconds. An image of the sample in the arc is provided in Figure 5. During the test, data was gathered successfully from

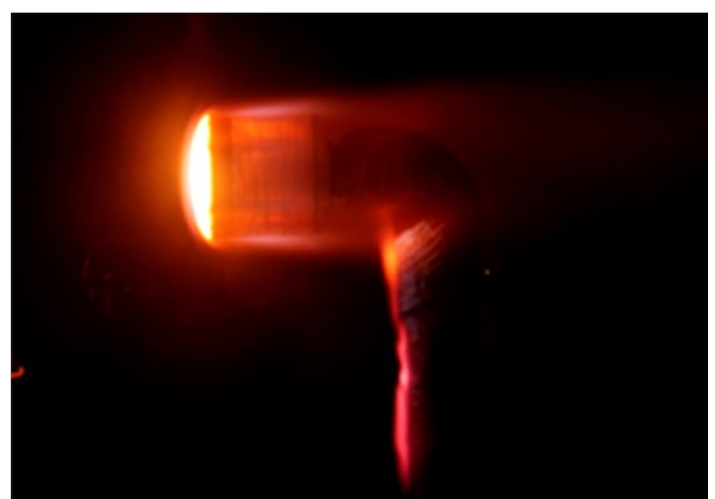

Figure 5. TPS sample with internal WLSS being heated in the AHF arc. one of the thermocouples, and that data was compared to data collected from that same thermocouple through a parallel hard-wired connection. The NASA hard-wired system was minimally impacted by this parallel connection because of the high impedance characteristics of the WLSS. Plots of the acquired data are shown in Figure 6.

\section{B. Discussion of AHF Test Results}

As seen in Figure 6, the real-time from the wireless, as well as the stored-and-forwarded wireless data (i.e., data stored in the WLSS onboard memory), at $2 \mathrm{~Hz}$ and 60 $\mathrm{Hz}$, respectively, closely track to the real-time wiredsystem data that were collected by the AHF's data acquisition system. Note that the wireless $2-\mathrm{Hz}$ and $60-\mathrm{Hz}$ data also map directly onto each other. An offset is visible between the NASA collected data and the WLSS data, as well as a break in the real-time wireless data between 15 and 75 seconds while the system was immersed in the arc plasma stream. The AHF test confirmed that a ZigBee network can exist near a high-density plasma environment and acquire temperature data.

The break in the data during the AHF test coincided directly with the period where the WLSS was encased within the plasma stream. The wireless signal was cut-off due to the plasma that forms around the system, a situation equivalent to radio black-out during re-entry. However, the system continued to operate during that exposure period, and instantly reestablished communication to the network as soon as the system was outside of the stream. This behavior demonstrated the viability of the ZigBee network for the WLSS. Additionally, since the wireless system is able to communicate during the period while the plasma jet is active but when the sample is no longer in the arc jet stream, an antenna placed at a location that is not encased in plasma would allow communication throughout a test. This configuration was tested during a subsequent test in the IHF.

The offset of the wireless with respect to the wired data is due to the lack of CJC to the thermocouple

\section{CUIP Test 2 WLSS/ WSS Comparison} Direct Data Comparison

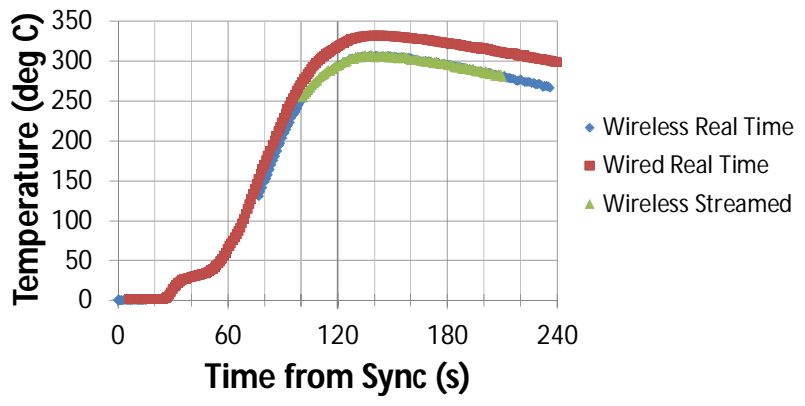

Figure 6. Comparison of data collected with wired and WLSS system in real-time and later streamed.

Wired and Wireless Real Time Slope

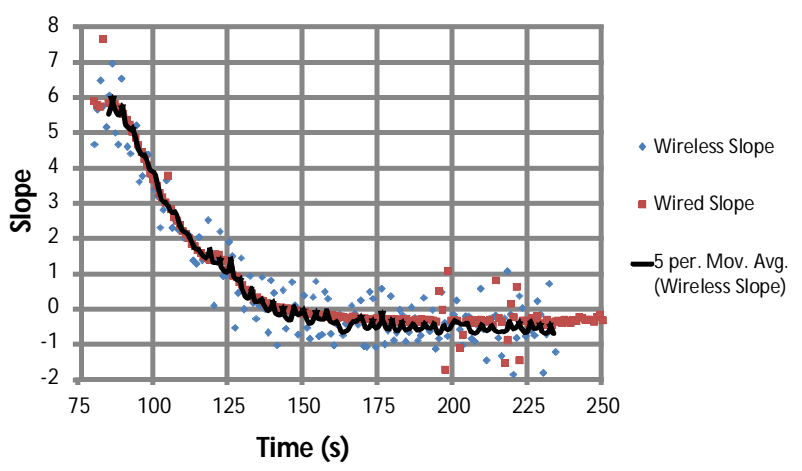

Figure 7. Wireless and wired real time slopes. wireless data; this offset becomes more pronounced at higher temperatures as it is a percentage of the value. This is shown by the similarity of the slopes (i.e., the time derivative of temperature) of the wired and wireless data as seen in Figure 7. Additionally, in Figure 8, the offset increases from 22 to $35^{\circ} \mathrm{C}$. This is attributed to the gradual heating 
of the sample inside of its thermal protection. In Figure 9, a test including CJC on the wireless system placed in a thermal chamber at Penn State showed correct mapping of the data from the temperature sensors. An LM35 temperature sensor was used to provide CJC for the thermocouple sensors, and these responses are shown to correctly reflect the actual temperatures.

The WLSS achieved $\pm 2.5^{\circ} \mathrm{C}$ accuracy with a single type-K thermocouple, with real-time (streamed) data rates of $2 \mathrm{~Hz}$, and high-speed data (stored onboard) collection rates of $60 \mathrm{~Hz}$ and above. The presence of the plasma within the chamber did not inhibit data acquisition, although there was a period of radio blackout while there was plasma between the system and the receiver

\section{WLSS Enhancements}

With the lessons learned from the AHF test, enhancements were made to the WLSS design with the following goals:

- Accommodate multiple sensor types

- Mitigate concerns regarding variations in facility preparation time and its impact on battery life

- Provide $100 \%$ real time data transfer at $2 \mathrm{~Hz}$ when the sample is in the flow

During arc jet sample testing, thermocouples, Gardon gauges, and pressure sensors are commonly used devices. These instruments are typically built into the test wedges used to evaluate arc jet test conditions. Each sensor differs in measurement voltage requiring separate processing chains for each, i.e., $0-5 \mathrm{~V}$ for the pressure sensor, $0-50 \mathrm{mV}$ for thermocouples, and $0-10 \mathrm{mV}$ for the Gardon gauge. Since the Gardon gauge and thermocouples produce voltages on the same order of magnitude, the WLSS uses similar signal conditioning paths, varying only in the required voltage gains. One of the channels previously allocated for thermocouples was used as a Gardon gauge input into a common instrumentation amplifier with selectable gain with a ratio of 5:1.

The pressure sensor required a more simple input circuit since its output signals are much larger in scale. The conditioning circuitry scaled and filtered the sensor output by half for compatibility with the $3.3 \mathrm{~V}$ system in the WLSS and a dedicated ADC. These two signal chains and the comparison with the original circuit can be seen in Figure 10.

In addition to modified signal conditioning and sampling circuitry, the team identified the need for greater flexibility in testing times citing the variability of setup time and facility debugging time. As we had demonstrated the system during the AHF tests running on battery power, we chose a wired power interface for IHF tests to ensure that the lengthier setup times for operations in the IHF would not drain

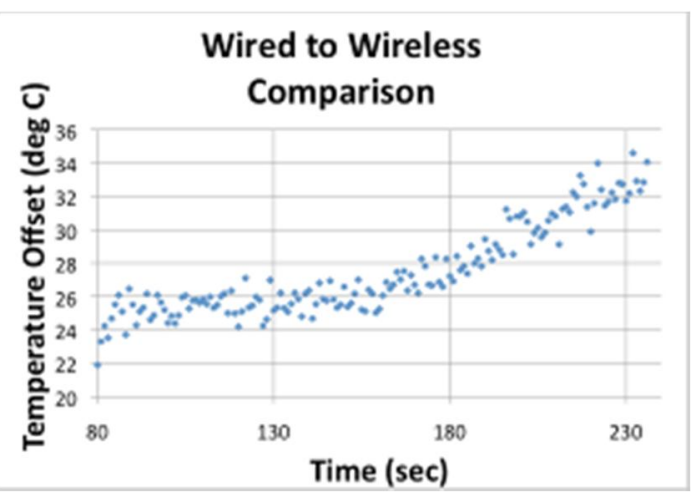

Figure 8. Offset between the wireless and wired data.

Figure 9. Cold junction compensation of the wireless system.

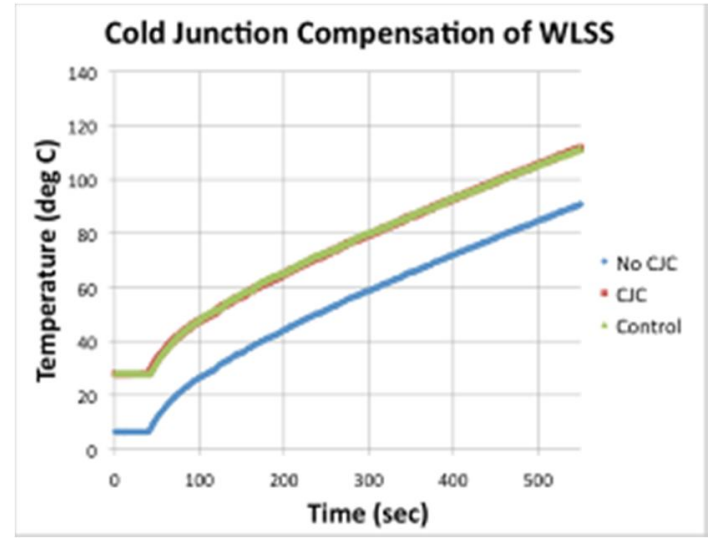

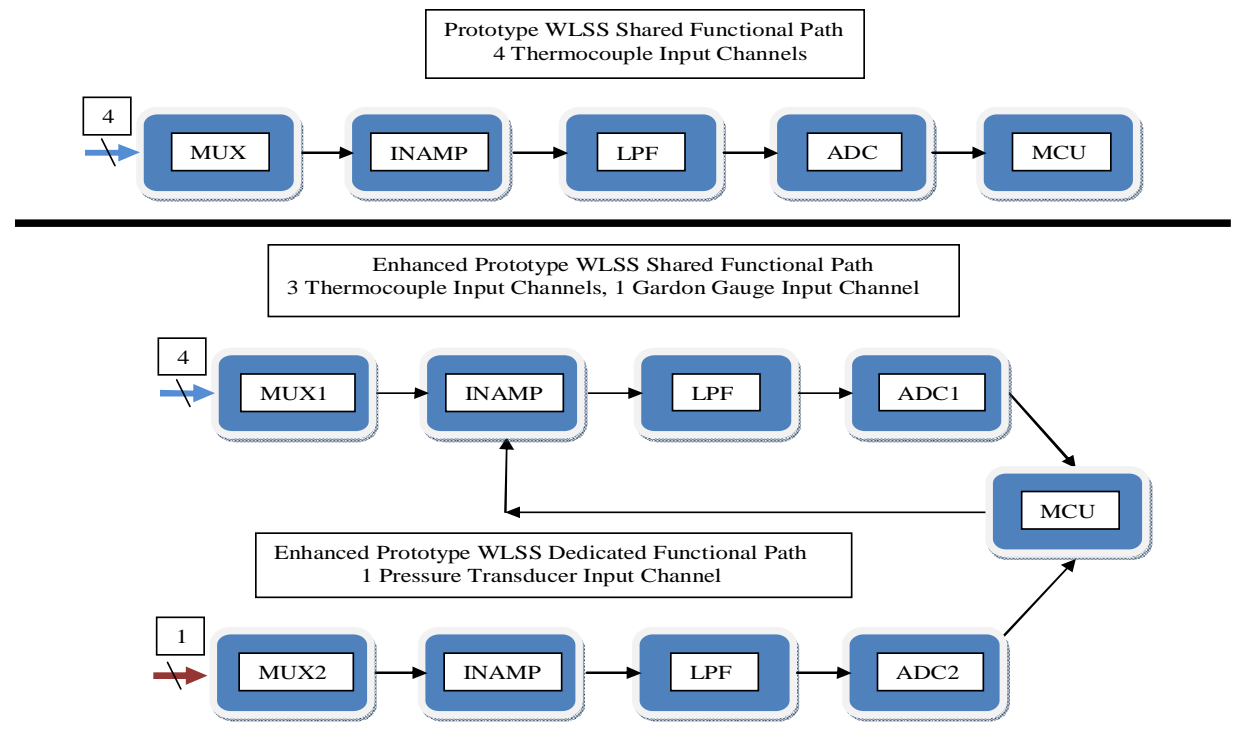

Figure 10. Enhanced prototype WLSS functional paths.

6

American Institute of Aeronautics and Astronautics 
the battery before testing could commence. Even with an externally wired power connection, employing a WLSS can still reduce overall system setup time by replacing the many wires per instrument with a single pair of wires through the sting arm.

\section{IHF Test}

With the enhancements made to the WLSS, another test opportunity was identified for February 2011 with the objectives of demonstrating additional measurement types and continuously transmitting $2-\mathrm{Hz}$ data throughout the test including when the sample is in the arc jet flow.

For testing within the IHF facility, the WLSS was integrated into the standard test wedge package in an enclosure mounted in place of the interconnect cover plate as shown in Figure 11. The top and front of the enclosure were covered with a LI900 tile for thermal protection. This configuration allowed easier access to the instrument signals with a minimal overall impact to the test function. For verification, the sensors were interconnected in a Y-junction configuration, thus passing the same signals to both the WLSS and facility sampling instruments as done in the

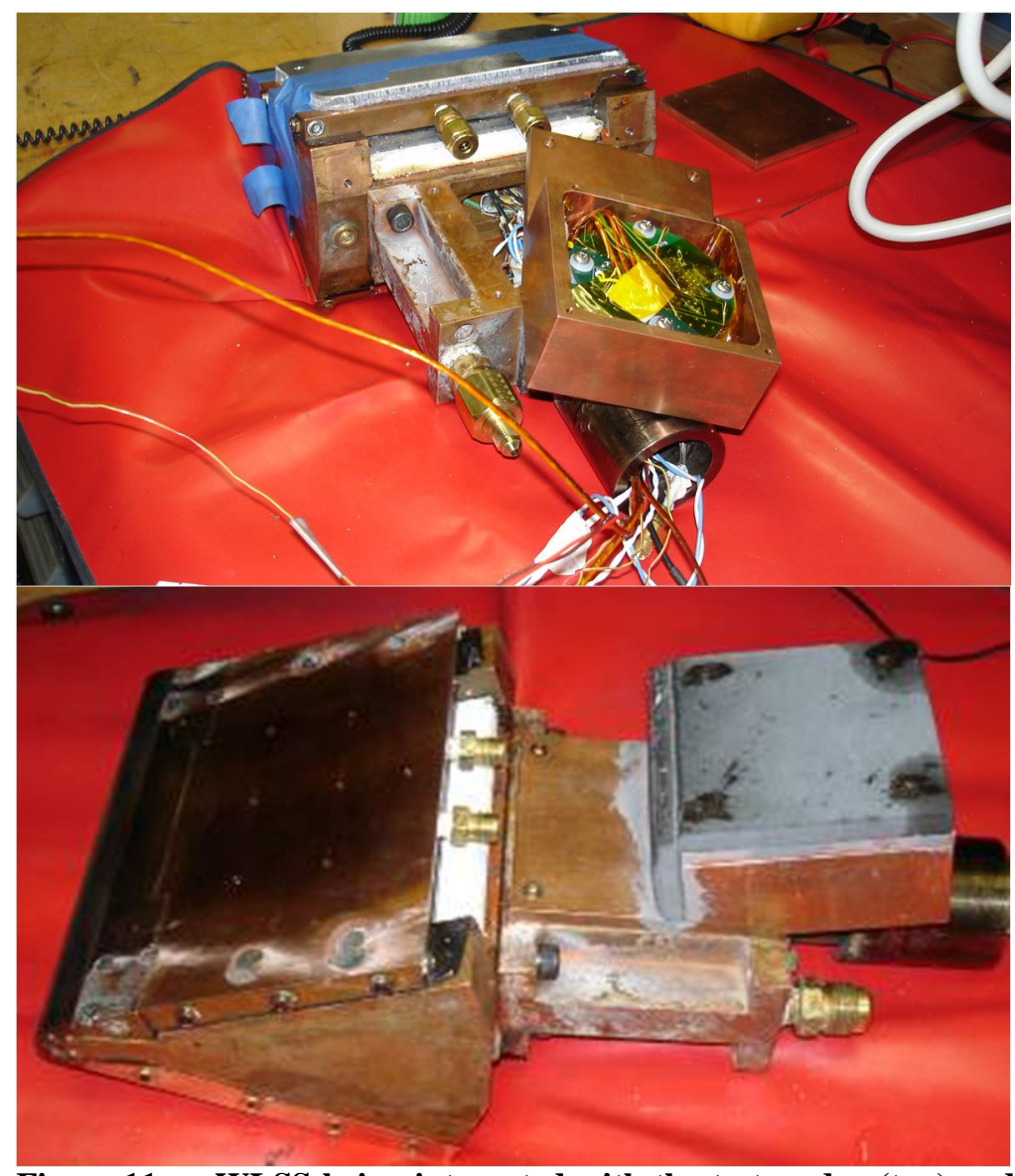

Figure 11. WLSS being integrated with the test wedge (top) and covered with thermal protective material (bottom).

previous AHF test. An external antenna was connected to the WLSS, passed through the sting arm, and placed at a location outside the region of expected plasma flow to allow wireless transmission of recorded data during the test (Figure 12).

\section{E. Discussion of IHF Test Results}

After installation of the wedge with WLSS but prior to the test, wedge coolant water leaked and entered the WLSS system. It should be noted that the need to be water-resistant was not captured in the prototype WLSS requirements, but are likely to be included in future revisions. After baking the system and treating sensitive sections with an alcohol wash, the low voltage thermocouple and Gardon gauge channels exhibited anomalous behavior when measuring steady-state temperatures and heat flux, and a dampening out of their response to faster signals such as those occurring during insertion to the flow. This was evident by good tracking of temperature data prior to

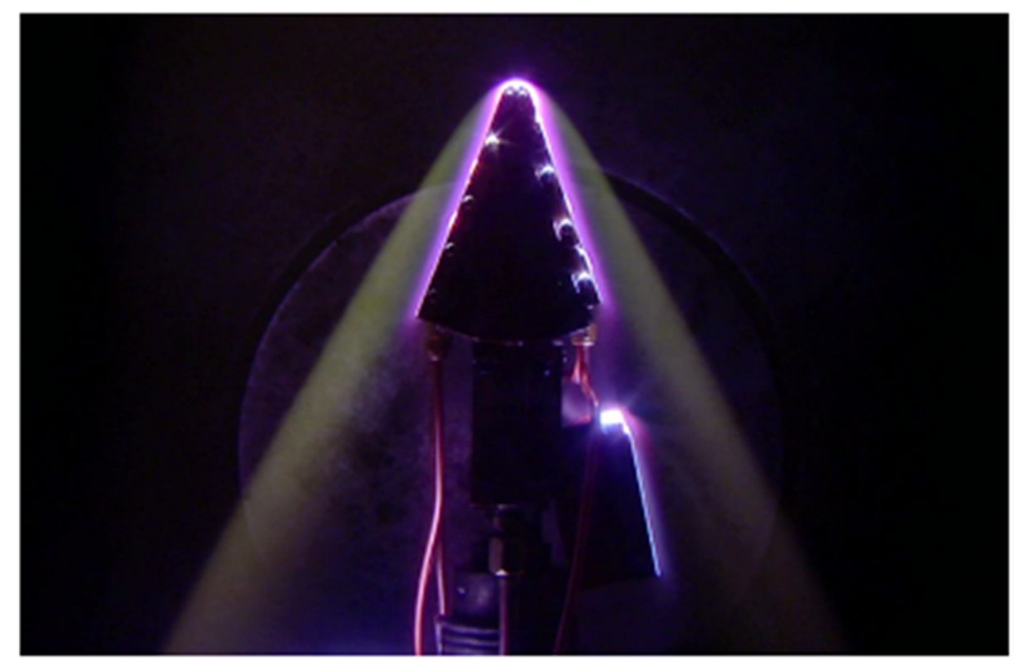

Figure 12. WLSS in the test wedge inserted into the flow from the IHF arc jet. the test but not during the actual insertion when a time-delay is seen in the circuit's response. The root cause of the delayed response is still under 
investigation. Over the course of four test insertions lasting 5 seconds each, the WLSS was able to achieve 100\% data transfer at the $2-\mathrm{Hz}$ real-time rate, demonstrating that, with the addition of an auxiliary antenna external to the flow, a continuous link can be maintained. During one of the test runs, data was received out of chronological order as can happen during ZigBee transmissions. All data, however, was received and their timestamps allowed the data to be placed in chronological order. Data was received in chronological order for the other three test runs.

The pressure system functioned as desired with test data shown in Figure 13. A $50-\mathrm{Pa}$ bias (equivalent to a $6-\mathrm{mV}$ bias) is present throughout this data set. In order to determine the root cause of this offset, several cause/effect scenarios were postulated, investigated, analyzed, and tested, including:

- Recalibration at room temperature

- Recalibration at $30^{\circ} \mathrm{C}$

- Calibration in a vacuum chamber at $\sim 2$

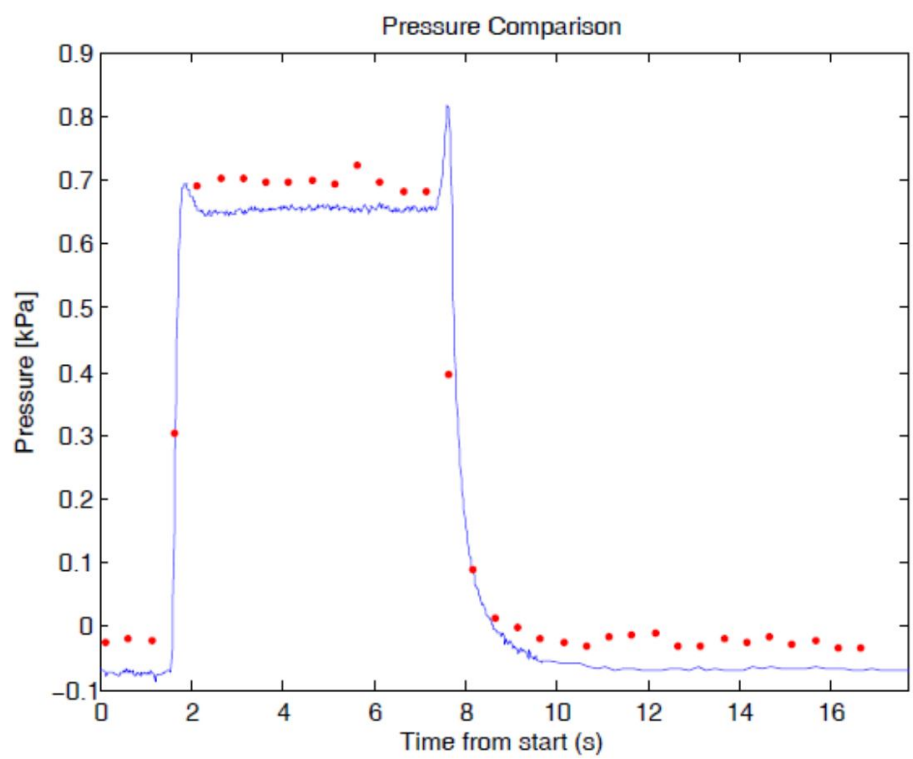

Figure 13. WLSS measured pressure in red compared with Arc Jet facility pressure in blue. 50 pa $(\sim 6 \mathrm{mV})$ bias is seen in the Torr

- Comparison of data prior to application of the high voltage in the arc jet heater.

The investigations showed unremarkable changes in the pressure calibration for the temperature and pressure conditions. Also, by comparing the pressure measurements before, during, and after exposure to the arc jet bus voltage, as depicted in Figure 14, no correlation of bias to bus voltage was evident. Although root cause of the 6$\mathrm{mV}$ bias was not determined, the experiments confirmed there is no correlation of bias to the significant and dominant environmental conditions, thus verifying the insignificance of the voltage offset, i.e., it can be removed with calibration.

\section{Conclusions}

The AHF and IHF tests validated the WLSS as a potential replacement for legacy wired systems. The AHF test was able to demonstrate the viability of high-speed data communication, although the non-real-time nature of this high-speed communication may slow the adoption of such a system. In addition, the AHF test demonstrated the robust nature of the network in its ability to immediately re-establish communication after the predictable plasma-induced radio blackout occurred. The IHF test demonstrated multiple sensor support and $100 \%$ connection time throughout the entire test duration with the addition of an external antenna. The arc jet tests validated the ability of the WLSS to provide rapid test bed

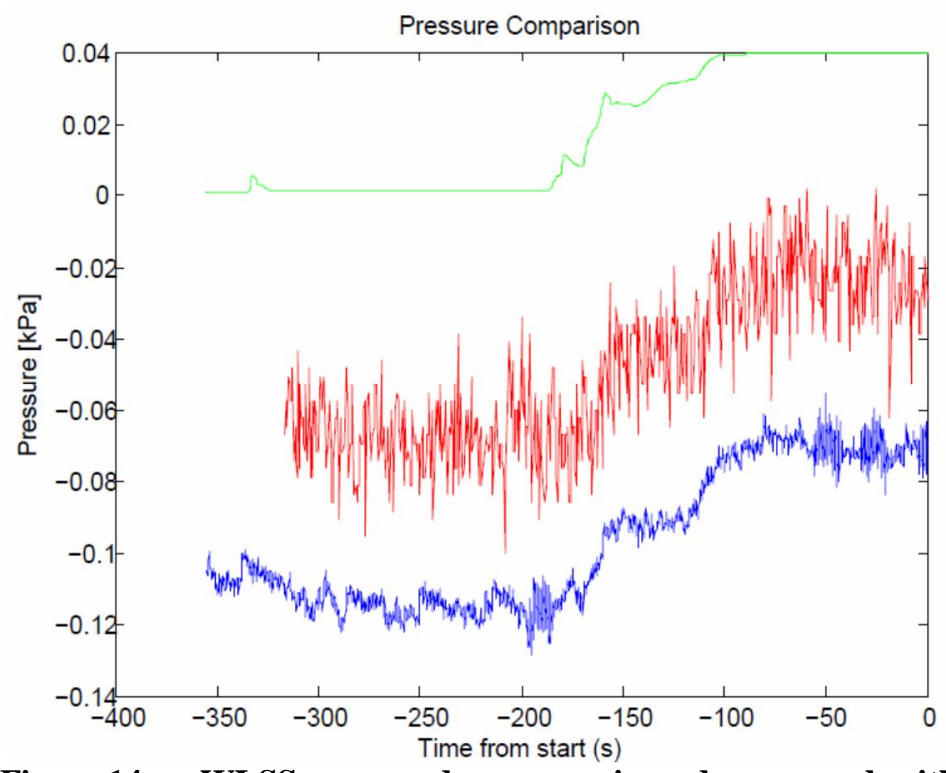

Figure 14. WLSS-measured pressure in red compared with arc jet facility pressure in blue. Time is in reference to the first insertion in seconds. Pressure values compared against the arc jet heater voltage waveform (green) for reference of effects of high voltage on measured signals.

reconfiguration and demonstrated compliant data acquisition without complex wiring and cabling systems. Additionally, the success of the AHF and IHF tests advanced the Technology Readiness Level (TRL) of the WLSS. 
In addition to the WLSS capabilities validated during AHF and IHF testing, the WLSS provides a reduction in the quantity and complexity of the physical interconnects, thus offering potential advantages over wired sensor systems (WSS) in several areas: reduced life cycle cost, improved reliability, increased accessibility, mass reduction, and adaptability. These WLSS advantages over the WSS apply to test facility applications as well as flight applications, thus highlighting the importance of and need for additional development to enable deployment of WLSS for both applications. Although there is commonality in the advantages offered to test facility and flight applications, future work for flight applications must include miniaturization of the system, optimization of data acquisition rate versus low power consumption, and development of wireless power systems.

\section{Acknowledgments}

We wish to thank B. Schratz for his work on system prototyping and design, K. Holmes for his high quality PCB assembling, and J. Georgiev for prototyping of the pressure measurement circuitry. We also acknowledge work at Univ. of Idaho by the ThermaSense and Thermal Exposure Capstone Design teams, which explored and tested single node and multiple node wireless sensor systems for TPS applications. Finally, we would like to thank the NASA CUIP program for providing the opportunity to pursue this research.

\section{References}

[1] Furse, C. and Haupt, R., "Down to the Wire," IEEE Spectrum, Vol. 38, No. 2, pp. 34-39, Feb. 2001.

[2] Furse, C., Chung, Y.C., Dangol, R., Nielsen, M., Mabey, G., and Woodward, R., "Frequency-domain Reflectometry for On-board Testing of Aging Aircraft Wiring," IEEE Trans. Electromagnetic Compatibility, Vol. 45, No. 2, pp. 306-315, May 2003.

[3] Atkinson, D., Swanson, G., and Schlee, J., "Implementation of Wireless TPS Sensors," Sixth International Planetary Probe Workshop, Georgia Institute of Technology, 21-27 June 2008.

[4] Bruning, A.M. and Campbell, F.J., "Aging in Wire Insulation under Multifactor Stress," IEEE Trans. Electrical Insulation, Vol. 28, No. 5, pp. 729-754, Oct. 1993.

[5] Lewis, F.L., "Wireless Sensor Networks," in Smart Environments: Technology, Protocols, and Applications, D.J. Cook and S.K. Das, editors, Wiley, 2004.

[6] "Our Members," ZigBee Alliance Home, ZigBee Alliance, Web, accessed $05 \quad$ Nov. 2010 <http://www.zigbee.org/About/OurMembers.aspx>.

[7] Mainwaring, A., Polastre, J., Szewczyk, R., and Culler, D., "Wireless Sensor Networks for Habitat Monitoring," ACM Workshop on Sensor Networks and Applications, 2002.

[8] Shan, Q., Brown, D., and Liu, Y., "Wireless Temperature Sensor Network for Refrigerated Vehicles," Proceedings of the IEEE International Symposium on Industrial Electronics, ISIE 2005, Vol. 3, pp. 1209-1214, 20-23 June 2005. 